THE IMPERIAL NATION 



\title{
The Imperial Nation
}

CITIZENS AND SUBJECTS IN THE BRITISH，FRENCH，SPAN ISH, AND AMERICAN EMPIRES

\author{
- \\ Josep M. Fradera \\ Translated by Ruth MacKay
}


Copyright (C) 2018 by Princeton University Press

Published by Princeton University Press

41 William Street, Princeton, New Jersey 08540

6 Oxford Street, Woodstock, Oxfordshire OX20 1TR

press.princeton.edu

All Rights Reserved

Library of Congress Control Number: 2018948275

ISBN 978-0-691-16745-9

British Library Cataloging-in-Publication Data is available

Editorial: Amanda Peery

Production Editorial: Kathleen Cioffi

Jacket Design: Amanda Weiss

Jacket Credit: (Top and bottom left) From Carte d'Europe, 1769.

(Bottom right) From Carte d'Amerique dressée pour l'usage du Roy, 1780.

Production: Erin Suydam

Publicity: Jodi Price

Copyeditor: Anne Cherry

This book has been composed in Miller

Printed on acid-free paper. $\infty$

Printed in the United States of America

10 98776543321 
To my son, Pere 
